\title{
Compte-rendu de la Conférence Internationale de Spéléologie et de Karstologie d'Istanbul (24 sept. -14 oct. 1964)
}

\author{
Par Paul Fenelon ${ }^{1}$ )
}

Avec 4 figures dans le texte

Au début de 1964, sous l'impulsion du Docteur Géologue Temucin Aygen, les karstologues et les spéléologues de Turquie décidèrent de créer une société nationale de Spéléologie. La richesse de leur pays en phénomènes karstiques, le souci des Services Publics de recueillir les moindres réserves d'eau pour l'irrigation et l'énergie électrique, enfin les activités scientifiques et sportives de jeunes explorateurs de plus en plus nombreux, justifiaient amplement une telle initiative. Pour inaugurer cette nouvelle Association savante, nos collègues turcs invitèrent à une Conférence Internationale les spécialistes du karst dans le monde entier. Des Français, des Allemands, des Espagnols, des Libanais, un Australien répondirent à leur appel et se trouvèrent réunis, au nombre d'une centaine, dans le grand amphithéâtre de l'Institut de Géographie de l'Université Beyazit, à Stamboul, le 24 septembre 1964.

L'accueil fut extrêmement chaleureux. Aidé par ses fidèles collaborateurs, Mesut Cetincelik, Cahit Sonmez, Adem Seber, Fahrettin Arslam, Ismaïl Yalcinlar, etc... Temucin Aygen avait soigneusement préparé la rencontre, de sorte que, grâce à un travail de plusieurs mois, il n'y eut aucune surprise désagréable dans le déroulement d'un programme minutieusement établi, où le confort matériel et l'intérêt scientifique ne connurent pas la moindre défaillance. Que nos aimables confrères de Turquie en soient félicités et remerciés.

La première séance s'ouvrit en présence du représentant de son Excellence Ali Isham Gogus, Ministre du Tourisme et de l'Informa-

1) Faculté des Lettres et Sciences Humaines de Tours (France). 


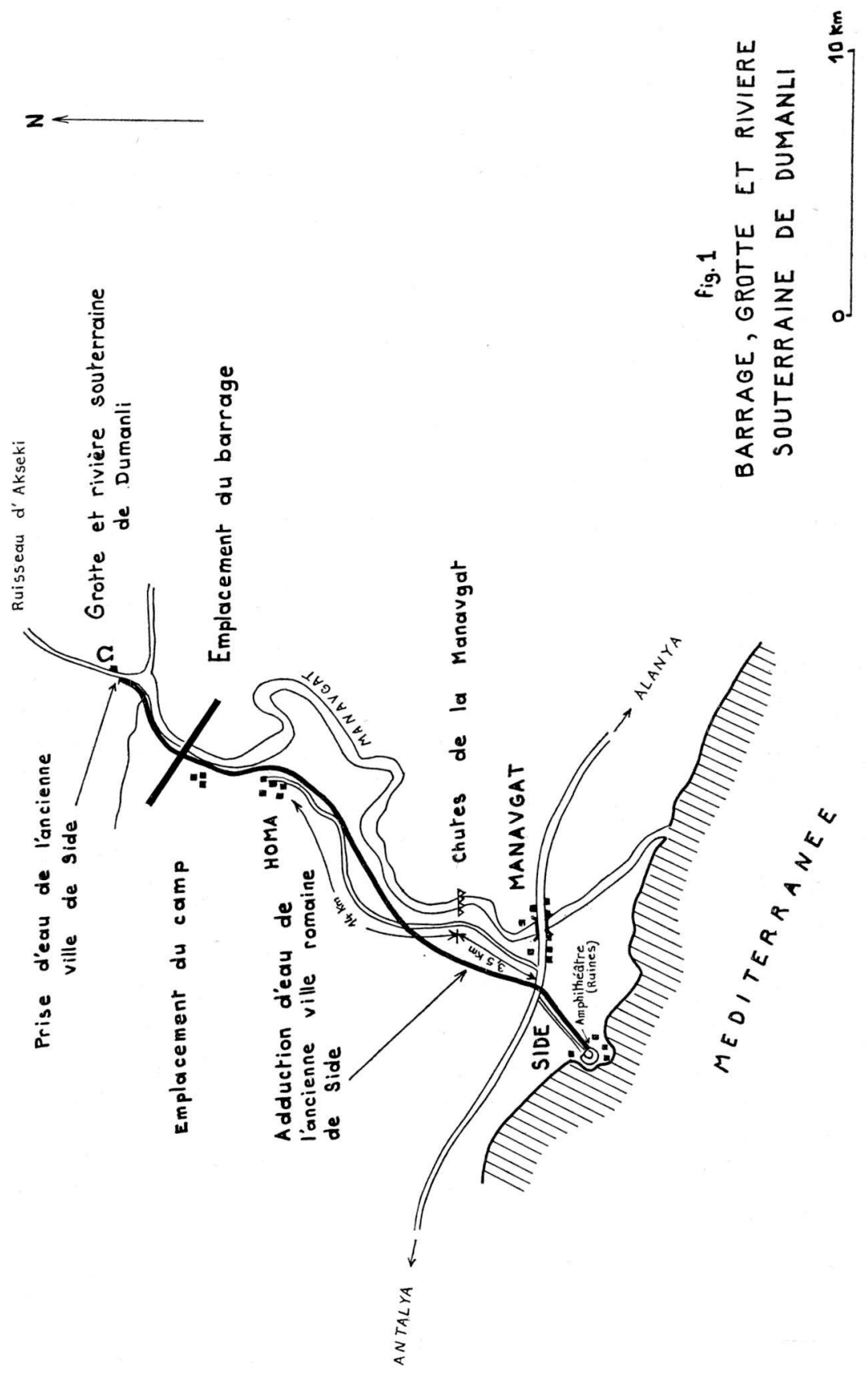


tion. Le Doyen en exercice de la Faculté des Lettres d'Istanbul, Mr. le Professeur de Philosophie Velpi Eralp, souhaita la bienvenue aux Congressistes en un français d'une pureté académique et en termes dont la délicatesse toucha vivement les savants étrangers venus participer à la Conférence. Au nom de ces derniers, le Professeur Fenelon, de l'Université d'Orléans-Tours (France), fit part aux organisateurs du plaisir et de l'intérêt qu'ils éprouvaient à se trouver dans la capitale prestigieuse de l'ancien empire ottoman.

Dans son discours d'ouverture, Mr. le Professeur Ahmet Ardel, Directeur de l'Institut de Géographie d'Istanbul, souligna les récents progrès de la Géomorphologie des régions calcaires grâce aux recherches effectuées, tant dans les laboratoires que sur le terrain, par les spéléologues et les karstologues. Puis, avec une amabilité souriante dont il ne se départit jamais au cours de notre séjour, malgré ses responsabilités et ses soucis, le Géologue Docteur Temucin Aygen, Président du Comité d'Organisation, évoqua le passé de la Spéléologie turque, signala les premiers résultats déjà obtenus par la jeune équipe qu'il a entraînée à l'exploration des grottes et des gouffres, et déclara ouverte la première séance de travail de la Conférence Internationale de Spéléologie d'Istanbul.

Les trois premières journées furent réservées aux communications des congressistes et à des excursions autour du Bosphore. Elles se terminèrent le samedi soir 26 septembre au cours d'une réception organisée par le Gouvernement turc que représentait Mr. le Directeur Général du Tourisme et de l'Information.

Du dimanche 27 septembre au mercredi 14 octobre, les membres du Colloque effectuèrent un voyage d'études à travers l'Anatolie, par Izmit, Ankara, Gorêmé, Konya, Eregli, Mersin, Alanya, Antalya, Burdur, Izmir, Bursa et Istanbul. Ils furent accueillis officiellement à Ankara par Mr. Ogüt Yazman, Directeur Général au Ministère de l'Information, à Antalya par les membres du Club local et à Izmir par le représentant du Ministère du Tourisme.

A notre grand regret nous ne pûmes, à cause de nos obligations professionnelles, suivre la seconde partie de l'excursion d'Antalya à Istanbul, par Izmir et Bursa. Néanmoins, ce que nous avions déjà vu de la Turquie au moment de notre retour était considérable. Nous avons rapporté de là-bas, sous forme de clichés, de cartes et d'ouvrages, une documentation extrêmement précieuse, d'où nous voudrions extraire, dans ce compte-rendu, un bref aperçu des travaux de la Conférence et des paysages karstiques d'Anatolie. L'intérêt scientifique du Colloque résida en effet aussi bien dans les séances de discussion qui se 
tinrent à Stamboul que dans les excursions qui se déroulèrent durant deux semaines à travers la péninsule d'Asie Mineure.

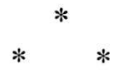

Dans le vaste amphithéâtre de l'Institut de Géographie de Beyazit, très bien équipé pour leurs travaux, les Congressistes entendirent une douzaine de communications relatives aux reliefs et à l'hydrologie karstiques. Au cours de la première, Mr. le Professeur Ismaill Yalcinlar évoqua successivement, dans un large tour d'horizon, les karsts du Nord et du Nord-Est de l'Asie Mineure, à Héraclée, sur la mer Noire et près d'Artvin, dans l'ancien pays de Lazes, puis ceux de la région de Karapinar, à l'Est de Konya, où des dolines circulaires, les "obrouks», sont taillées dans les calcaires néogènes subhorizontaux reposant en discordance sur les terrains permo-carbonifères vigoureusement plissés. Quelques-uns de ces obrouks, fossilisés par les dépôts volcaniques de la fin de l'ère tertiaire, ont été remis en valeur par l'érosion des temps quaternaires. Mais c'est au Sud de l'Anatolie, dans les calcaires du Jurassique et du Miocène moyen, que les phénomènes karstiques sont les plus fréquents. Dans les anciennes provinces de Cilicie, de Pamphylie et de Lycie, le socle hercynien s'infléchit vers le Sud, sous l'influence, semble-t-il, de la flexure continentale. Avec lui, sa couverture, composée surtout de calcaires lacustres tertiaires, s'incline vers la Méditerranée, toute perforée de dolines et coupée de cañons comme celui du Göksu. L'auteur fit un sort particulier au poljé de Mugla, en Lycie, où se perd la rivière Ciné avant de pouvoir atteindre le Méandre ; il évoqua également celui de Kestel qui correspond au sommet d'un anticlinal.

Cette dernière remarque permit au Professeur Ahmet Ardel de souligner les analogies entre le karst dalmate et celui du Taurus occidental. De part et d'autre, la tectonique, l'érosion normale et la corrosion ont joué de concert pour donner, durant la fin du Pliocène, des poljés fort complexes; la plupart sont liés à des accidents tectoniques; celui de Kestel est en fait une combe ; celui de Mugli correspond à des failles SW-NE. Des strates de flysch, intercalées entre celles du calcaire, viennent encore compliquer les reliefs de surface et les cavités souterraines par leur insolubilité et leur imperméabilité, de sorte qu'il ne faut pas perdre de vue, dans l'explication de cette géomorphologie karstique, la convergence de plusieurs influences géologiques.

En outre, pour clore la discussion, Mr. Aygen attira l'attention sur les karsts encore mal connus d'Héraclée, de Filyos et d'Akçabat près de la mer Noire. De même les régions situées dans le coude de l'Eu- 
phrate vers Elazig mériteraient de longues investigations afin d'utiliser au maximum les ressources hydrauliques d'une région semiaride.

Un jeune Assistant de la Faculté des Sciences de Montpellier, Mr. Claude Drogue, disciple de Mr. le Professeur Avias, nous fit part, au cours d'une seconde communication, de ses recherches sur les résurgences qui donnent naissance à la Sauve, au Lirou et au Lez dans les plateaux calcaires des Garrigues montpelliéraines. Par l'analyse des eaux, et avec de la fluorescéine, il est parvenu à établir l'origine souterraine des sources qui sont liées, tout au moins les deux premières, à des lignes de failles. En intégrant pluies anciennes et pluies récentes, pente du relief, vitesse de ruissellement et résistance des roches et de la végétation à la circulation des eaux, à l'aide de hyétogrammes et de bandes d'écoulement isochrones, il a dressé pour ces rivières des hydrogrammes de crue et de décrue. En particulier, les courbes de décrue mettent en évidence de grandes différences entre les trois cours d'eau; les décrues du Lirou durent jusqu'à 8 jours, celles de la Sauve jusqu'à 10 jours et celles du Lez ne s'atténuent qu'après une quarantaine de jours. Dans sa conclusion, Mr. Drogue souligne l'intérêt des hydrogrammes, correctement établis et finement analysés, pour établir les lois de l'écoulement des eaux dans les roches calcaires. Si nous en jugeons par les tirés à part qu'il nous a remis, notre collègue de Montpellier a devant lui une œeuvre de longue haleine; mais elle sera fertile en résultats concrets, grâce à l'utilisation des ions naturels en solutions comme traceurs dans les aquifères calcaires des karsts sous-cévenols. Déjà en sont la preuve ses essais de délimitation du bassin d'alimentation d'une résurgence dans les karsts noyés du Languedoc ${ }^{2}$.

Le Professeur Fenelon, Président de la Commission des Phénomènes Karstiques du Comité National Français de Géographie, présenta aux membres de la Conférence une Introduction destinée à un Vocabulaire des principaux termes utilisés en France pour désigner les accidents aériens et souterrains des karsts. Au cours de son intervention, il indiqua les origines de ce Vocabulaire, l'aide que lui ont apportée plusieurs collaborateurs, et il souligna l'utilité de telles publications en plusieurs langues pour permettre des comparaisons exactes d'un pays à l'autre. Sa communication sera publiée dans les Actes de la Conférence et fera partie, avec le Vocabulaire, d'un Volume des Mémoires et Documents du Centre National de la Recherche Scientifique à Paris, ouvrage qui sera consacré aux reliefs karstiques.

2) Annales de Spéléologie, Tome XVIII, fasc. 4, 1963, P. 405-414. 
Les obrouks, déjà signalés par Mr. le Professeur Yalcinlar, donnèrent lieu à une communication fort pertinente de l'hydrogéologue Adem Seber. Ces gouffres, étudiés dans la région de Konya, ont des diamètres très variables, de 100 à $300 \mathrm{~m}$., et des profondeurs qui peuvent atteindre une centaine de mètres. Creusés dans des calcaires lacustres néogènes, ils paraissent dus à des phénomènes concomitants de corrosion et d'effondrement. Un lac paraît dormir au fond de ces obrouks, retenu par le substratum imperméable, marnes ou schistes cristallins sur lesquels se sont déposés les calcaires tertiaires. En fait ses eaux sont pures ou troubles selon que règnent la sécheresse ou les pluies. Elles s'écoulent donc selon un sens que Mr. Adem Seber a mis en évidence en mesurant la température de ces eaux au pourtour de ces gouffres et en la comparant à celle de l'air. Quand l'eau des lacs a une température plus élevée que celle de l'air, le courant s'établit des températures les plus hautes vers les températures les plus basses; c'est le contraire si les eaux sont plus froides que l'air. Ainsi, on a pu déceler un déplacement très lent dans les lacs des obrouks, si lent que nul moulinet ne pouvait le mettre en évidence.

Par de savants calculs, l'hydrogéologue Adem Seber a jaugé le volume des eaux contenues dans l'obrouk de Kizoren $\left(6 \times 10^{6} \mathrm{~m} .{ }^{3}\right)$ et dans celui de Timras $\left(1,3 \times 10^{6} \mathrm{~m}^{3}\right)$ afin d'apprécier la quantité que l'on peut prélever pour l'irrigation sans assécher ces réservoirs naturels. Pour le premier, elle atteindrait 95,4 1./sec. pour un abaissement de $10 \mathrm{~m}$., et pour le second 147 l./sec. pour le même abaissement. Ces données montrent que l'on pourrait ainsi irriguer 2780 ha. de terre. Des échelles limnimétriques ayant été installées dans ces deux gouffres, les variations du niveau de l'eau dans le lac Kizoren sont en retard de deux mois sur les pluies ou la sécheresse, tandis qu'elles sont presque instantanées dans le lac de Timras. On ne sait pas encore à quoi tiennent ces différences de durée.

Avec beaucoup de brio, de netteté et d'entrain, Mme. Muzaffer Turkman, Membre de la Direction des Eaux d'Ankara, présenta en français, puis en turc, le résultat de ses observations sur la géochimie des eaux karstiques en Turquie. Après avoir effectué des mesures sur divers calcaires prélevés en plusieurs régions et obtenu des pourcentages de $\mathrm{CO}_{3} \mathrm{Ca}$ variant de 80,7 à 98 , elle a procédé à des dosages d'eaux issues de ces masses calcaires et elle a abouti à des conclusions mises en valeur par des diagrammes semi-logarithmiques et triangulaires. Ainsi, au cours de cinq prélèvements, dont le $\mathrm{pH}$ variait entre 7,7 et 8,1 , ce qui représentait tout au plus 2 à $3 \mathrm{mg}$. de $\mathrm{CO}_{3} \mathrm{Ca}$ par litre, la Karstologue s'est aperçue que ces eaux étaient également chargées de chlorure de sodium et de matières organiques, ce qui les 
rendait impropres à l'alimentation et même à l'irrigation. Les observations de Madame Turkman aboutissent done à des solutions pratiques, fort utiles dans un pays où la recherche de sources abondantes est, comme nous l'avons dit, l'une des principales préoccupations des pouvoirs publics.

L'utilisation des radio-isotopes, pour déterminer l'origine des sources vauclusiennes et pour mesurer les débits des cours d'eau, fournit au jeune géologue Mesut Cetincelik l'occasion de montrer l'intérêt que présentent, pour ces expériences, les produits dont dispose le Commissariat à l'Energie Atomique (C.E.A.) de France. Avec un personnel entraîné à ce genre de recherches, les isotopes radioactifs remplacent avantageusement les anciens procédés pour déterminer les caractères hydrogéologiques d'un karst. En particulier, on peut apprécier d'une manière trés précise, non seulement l'origine des résurgences, mais également leurs débits et leurs régimes, à condition toutefois d'avoir le matériel approprié pour effectuer de délicates mesures.

Mr. Cl. Pommier, Conseiller administratif du département de la Drôme, et dont le violon d'Ingres est l'exploration des grottes du Vercors, décrivit aux Congressistes d'Istanbul celle d'où sort la Luire, affluent de la Bourne. Creusée dans les calcaires urgoniens, ses sinuosités, de section circulaire ou ovale, s'expliquent par le jeu des diaclases et des joints de stratification. Des courants d'air permanents y agitent l'atmosphère et produisent des ronflements quand le vent est violent. Elle devient très dangereuse par temps de pluie prolongée à cause des engorgements dus à l'afflux trop abondant et parfois subit des eaux de ruissellement.

A la suite de cet exposé, le Délégué de la Fédération française de Spéléologie nous présenta un matériel moderne pour l'exploration des grottes : canot pneumatique, échelle de nylon et de fil de fer léger, mais très résistant, crampons d'acier durci, matériel qui retint vivement l'attention des spéléologues du colloque. Enfin, Mr. Cl. Pommier termina par la projection d'un très beau film réalisé durant l'exploration du gouffre Berger.

Deux tirés à part, l'un des Annales de Spéléologie, T. XVI, fasc. 3, 1961, p. 227, et l'autre des Mémoires de Spelunca, $\mathrm{N}^{\circ}$ 2, 1962, p. 54, dus à la plume de MM. Garnier et Pommier, permirent aux auditeurs de préciser certains détails de la claire et solide conférence de Mr. Pommier sur la grotte de la Luire où notre collègue a atteint la cote 350 et exploré $8 \mathrm{~km}$. de réseau; seuls les brusques changements de niveau des eaux l'ont empêché d'aller plus loin et plus bas.

La communication de l'organisateur du colloque, le Géologue Temucin Aygen, fut consacrée aux recherches d'eau pour l'irrigation. 
Après avoir souligné la pauvreté en précipitations atmosphériques de la plupart des contrées de l'Anatolie, particulièrement en été durant la période végétative, le conférencier décrivit certains travaux effectués pour accumuler des réserves importantes derrière les barrages, parfois avec succès, parfois avec échec, comme cela se produisit en 1960 dans le réservoir de May, près de Konya ; l'eau s'y éleva de 4 m., puis elle disparut par des avens qui s'ouvrirent dans la masse d'argile colmatant le fond de la vallée. Si les services publics avaient consulté les hydrogéologues, ils auraient évité cette déception.

Grâce à sa compétence en karstologie, Mr. Temucin Aygen a donné en plusieurs occasions d'utiles conseils aux ingénieurs des Travaux Publics, en particulier à Antalya où, comme nous le verrons au cours de nos excursions, il a redressé une situation compromise par la construction d'une usine électrique qui avait privé d'eau, pour l'irrigation de leurs champs, les agriculteurs de la région. Dans le bassin houiller d'Héraclée (Eregli), il a tracé des lignes de sécurité autour des sources qui alimentent les usines et qui paraissaient menacées par le creusement de carrières dans les calcaires viséens. La Société des Houillères craignait en effet que les coups de mine fassent dévier les cours d'eau souterrains. Mais les précautions ayant été prises, on peut extraire à $5 \mathrm{~km}$. des hauts fourneaux et non à 12 , le calcaire indispensable à l'industrie sidérurgique d'Eregli.

Par contre, à Kayzerieh, des hydrauliciens, qui ont mené une enquête destinée à augmenter le débit des aqueducs alimentant la ville en eau potable, faute de quelques conseils, n'ont pas su éviter un fâcheux affaissement de terrain auprès de la centrale électrique. C'est également vers le Sud-Est de l'Anatolie, en direction de la Djéziré, que les recherches en eau d'irrigation seraient souhaitables. Les sources de type vauclusien n'y font pas défaut. C'est par une étroite collaboration entre géologues et spéléologues que l'on parviendra, dans ces régions arides, à accumuler de l'eau pour étendre les terres irrigables. Tel est le souhait du Président de la nouvelle Société de Spéléologie turque. Les membres du Congrès ne purent que s'y as socier et applaudir l'éminent orateur.

L'une des dernières interventions, celle du Professeur Jennings, de l'Université de Canberra, nous fit effectuer par la pensée un tour d'horizon complet à travers les régions karstiques d'Australie. Après un rapide compte-rendu historique des recherches passées, où furent citées en particulier celles de Danes et de S. W. Carey, le conférencier décrivit les massifs calcaires soulevés et basculés des Alpes australiennes, les «turmkarsts» du Queensland tropical, les sédiments récifaux du Nord-Est, pincés dans les blocs du socle; et enfin les immenses 
plaines de Nullarbor, perforées de dolines d'effondrement. Reprenant un sujet traité à Settle, lors du symposium qui suivit le Congrès international de Géographie de Londres, le savant géographe australien analysa la formation des phénomènes karstiques dans les dunes littorales riches en carbonate de calcium. Pour étayer ses conclusions, tout en ajoutant de l'agrément à ses propos, le Professeur Jennings fit défiler sur l'écran une remarquable série de diapositives provenant de son pays d'origine.

Entre temps, et pour nous donner un avant-goût de ce que nous allions pouvoir observer au cours de notre randonnée à travers l'Anatolie, Mr. Temucin Aygen avait projeté et commenté des clichés de grottes, de cavernes, de résurgences et de cascades situées dans les régions calcaires du centre et du sud de l'Asie Mineure, aussi bien sur les hautes plaines de Cappadoce que sur les rivages de la Pamphylie et de la Paphlagonie.

A midi, le samedi 26 septembre, le Président de la délégation française, qui avait dirigé les débats de la dernière séance, prononçait le discours de clôture de la première partie de la Conférence, en soulignant combien les diverses communications, et les discussions qu'elles avaient suscitées, avaient permis aux karstologues d'une dizaine de pays de confronter avec profit leurs observations et leurs conclusions relatives aux phénomènes karstiques.

Un seul, mais très profond regret affligea les Congressistes, ce furent les absences inattendues du Professeur Motas, Conseiller Scientifique de l'Institut de Spéléologie de Roumanie, et de Mr. Kuchta Gijula, Conservateur de la Bibliothèque Centrale de Budapest. Le premier devait nous entretenir des recherches phréatobiologiques en Roumanie; le second avait proposé une communication sur les réalisations pratiques de la Spéléologie en s'appuyant sur les méthodes et sur la collaboration des explorateurs de grottes, sur la signification et le rôle de l'étude des cavités souterraines et sur leur description scientifique. Pour des motifs qui n'ont aucun rapport avec les préoccupations ordinaires des karstologues, ces deux savants ne purent se rendre à Istanbul. Mais les Congressistes, tout en déplorant leur absence, leur adressèrent un salut confraternel et un témoignage de chaleureuse admiration pour le rôle éminent qu'ils jouent dans le domaine de la karstologie.

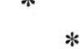

Le voyage d'études qui fit suite aux conférences d'Istanbul et qui se déroula des rives du Bosphore aux plateaux d'Ankara et de Konya jusqu'aux rives méridionales de l'Anatolie et à la façade occidentale 
de l'Asie Mineure sur la mer Egée, permit aux Congressistes d'admirer les récentes réalisations urbaines de la capitale turque, les ruines laissées par les brillantes civilisations hellénistiques, byzantines et seldjoukides, et ils purent également observer des contrastes saisissants entre les anciens modes de vie où se conservent de pittoresques traditions et les créations actuelles des industriels, des agriculteurs et des administrateurs formés à l'école du prestigieux Mustapha Kemal Ataturk. Pour sa part, la délégation française n'eut pas de peine à déceler, au cours des conversations et des réceptions, les liens qui unissent les universitaires de France et les milieux cultivés de la Turquie. Elle ne put que regretter le faible rôle que joue encore notre pays dans le domaine de l'équipement matériel de la jeune république.

Néanmoins, le principal but de l'excursion organisée par Mr. Temucin Aygen résidait dans l'examen des phénomènes karstiques. Sans doute géologues et géomorphologues s'intéressèrent-ils aux reliefs volcaniques d'Aksaray et de Karapinar, ainsi qu'aux étranges paysages découpés par une érosion violente dans les tufs volcaniques d'Urgüp et de Gorêmé. Toutefois, c'est vers les formes topographiques propres aux calcaires : lapiés, dolines, grottes et sources vauclusiennes que s'orientèrent le plus souvent les longs et fructueux cheminements du voyage. En fait, l'examen des phénomènes karstiques ne débuta qu'autour de Konya et ne prit toute son ampleur que sur le littoral méditerranéen. En effet, comme nous l'avons déjà signalé, les massifs calcaires sont rares au Nord et au Centre de l'Anatolie. Il faut atteindre le Taurus pour en découvrir d'innombrables exemples. Cette jeune chaîne de montagne, qui s'étend de la Lycie au coude de l'Euphrate, comporte des hauts plateaux et des contreforts composés de calcaires datés du Permien, du Jurassique, du Crétacé et, en bordure de la Méditerranée, du Miocène moyen et inférieur. Ce furent surtout ces derniers qui retirent notre attention entre Konya et Mersin, et vers Antalya, dans une zone d'ensellement qui affecte les ondulations du Taurus, et dans la pincée qui fait se rebrousser les plis de part et d'autre du lac d'Eregli et du fleuve Akçay. Ces calcaires tertiaires bordent la région littorale de Mersin à Antalya, sauf de Silifke à Antalya où les roches sédimentaires du socle ancien dominent les flots. Ce sont également de part et d'autre d'Anamur, des sédiments riches en carbonate de calcium qui favorisent grottes et pertes de rivières. De sorte que notre documentation sur les reliefs karstiques ne cessa de s'enrichir de Konya à Antalya ${ }^{3}$ ).

3) Voir I. Yalcinlar: Quelques observations sur les régions karstiques de Turquie. Review of the Geographical Institute of the University of Istanbul. $N^{\circ} 9-10,1964$, p. 147. 
Sans doute, à cause du temps trop limité, né pûmes-nous pas traverser les hautes régions karstifiées du Sakaralayadag, aux sources du Lamos, près de la route de Kasaman à Silifké, régions toutes trouées de dolines et de gouffres encore mal explorés. Il nous fut également impossible d'atteindre les hauteurs du Kizilgagh, au Sud d'Ermenek et de Kazanci, les routes qui auraient pu y donner accès étant encore de parcours difficile. De même, obligés d'interrompre le circuit à Antalya, nous dûmes laisser aux autres membres du Colloque le soin et le plaisir de visiter au Sud-Est de Burdur les sources de Gökpinar, la grotte d'In et les travaux qui ont abouti à l'équipement de la centrale hydroélectrique mise en œuvre par les eaux de l'émergence destinée à alimenter Burdur en courant. La grotte de Kali, riche en gisements préhistoriques, nous aurait également tentés au Nord d'Alanya, si la nécessité de suivre un itinéraire soigneusement tracé ne nous en avait écartés. Nous ne pouvions pas non plus former le projet d'aller à l'Ouest du lac de Beyschir visiter la grotte de Pinargözu, récemment explorée par Mr. Temucin Aygen. Il en sort une rivière très abondante au printemps et qui s'écoule vers Beyschir, non sans se perdre deux fois dans les calcaires du Crétacé supérieur.

Mais ce que nous vîmes en huit à dix jours fut cependant amplement suffisant pour nous donner une idée très détaillée de la diversité des

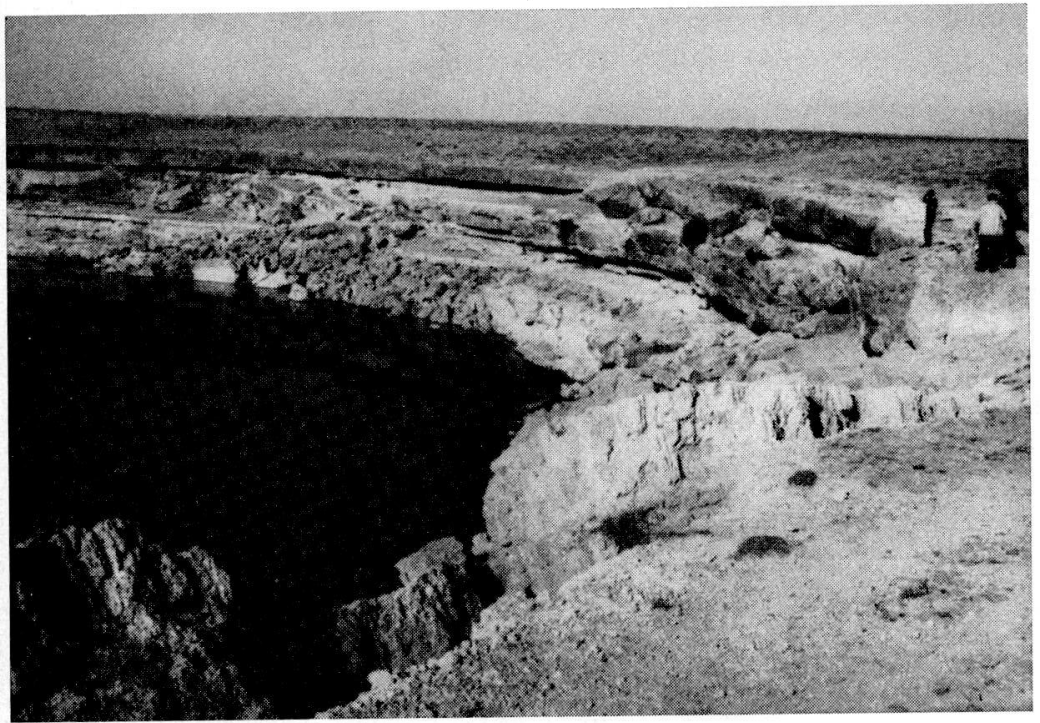

Fig. 2. «Obrouk» dans les calcaires miocènes. 
karsts dans l'Anatolie centrale et méridionale. Voici, au fil des kilomètres, quelques-unes des notes prises au cours de notre randonnée de Konya à Antalya. A une cinquantaine de kilomètres de la vieille cité, près de la localité de May, sur l'un des cours d'eau qui descendent du massif de l'Alacadag pour aller se perdre dans les calcaires miocènes et les dépôts quaternaires du bassin de Cumra, on a construit en 1959 un barrage destiné à l'irrigation des plaines voisines. Mais pour édifier le mur, on retira du fond du lit mineur du cours d'eau des masses d'argile qui colmataient un substratum calcaire. Les conséquences de cette fausse manœuvre ont entrainé la disparition des eaux de ce réservoir dans les anfractuosités de la roche. Elles vont rejoindre sans utilité les nappes souterraines qui alimentent le grand Tuz Gölu. Si les services publics avaient demandé l'avis d'un spécialiste des phénomènes karstiques, peut-être auraient-ils évité cette mésaventure ; peutêtre n'auraient-ils jamais entrepris un barrage aussi malencontreusement situé.

Une autre randonnée au Sud-Est de Konya amena le groupe des karstologues autour d'un obrouk percé à l'emporte-pièce dans la couverture subhorizontale des calcaires miocènes. Il s'agit d'une cavité circulaire de près de $200 \mathrm{~m}$. de diamètre et d'une cinquantaine de mètres de profondeur, entourée d'escarpements à pic. Au fond semble dormir un lac d'eau douce, claire et calme (Fig . 2). En fait, elle s'écoule lentement du Sud vers le Nord selon la pente du socle sur lequel reposent les strates calcaires. C'est sans doute un cours d'eau souterrain, favorisé par la corrosion et les infiltrations de ruisselets dont on aperçoit les ravins autour de la cavité, qui a provoqué par soutirage l'effondrement progressif de la voûte d'une caverne. A cause de la pente générale des strates vers le Nord, les bords de l'obrouk sont légèrement dissymétriques.

A travers une région très tourmentée, où alternent les barres rocheuses du Jurassique et du Crétacé et les massifs calcaires percés d'intrusions ophitiques, le car nous transporta le mercredi 30 septembre, à Yerköprü, dans les gorges que le Göksu a creusées à travers le massif du Kartaldag. Dans cette gigantesque coupure, due à l'ampleur de la montagne et à la proximité du niveau de base méditerranéen, le fleuve a déposé, en plusieurs niveaux de 400 mètres d'épaisseur, des lits successifs de travertins. Les eaux du Göksu sont encore si riches en calcite qu'elles pétrifient en quelques semaines les feuillages qui, au printemps, poussent à travers elles. Cependant la masse de ces dépôts est telle qu'il faut envisager un climat plus humide et un cours d'eau coulant 400 mètres plus haut qu'actuellement et s'encaissant progressivement, pour expliquer une telle accumulation de travertins. 
En outre, on comprend mal pourquoi ces travertins se sont formés en ce point de la vallée et continuent à s'y former, plutôt qu'en amont ou en aval. Peut-être y avait-il là une abondante végétation, favorisée par la présence de sédiments tertiaires au sol fertile. A travers ces barrages de plantes diverses, des eaux très dures se divisaient et se brisaient, laissant se déposer la calcite sur les rameaux des plantes. Actuellement, ces mêmes eaux, qui ont déblayé d'énormes quantités de travertins anciens, coulent en amont au niveau des plus récentes strates de calcite; elles y pénètrent par de nombreuses anfractuosités et vont se briser en multiples cascatelles dans des grottes sombres et humides (Fig. 3). A moins d'un kilomètre vers l'aval, le Göksu, grossi par les sources vauclusiennes de Karasu, est capté dans un canal qui

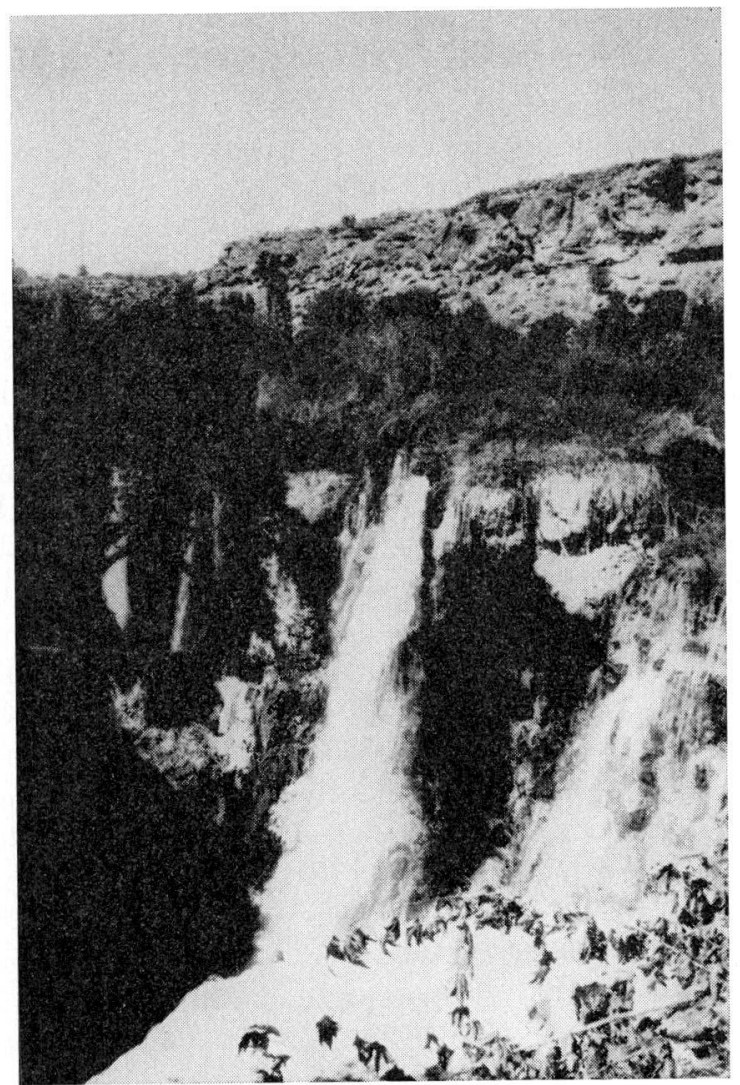

Fig. 3. Cascade du Göksu dans les travertins de Yerköpru. 
le conduit au-dessus de la centrale hydroélectrique de Yerköpru. Cette centrale alimente en courant, par une ligne aérienne de transport de force, la ville de Konya.

C'est également une très abondante émergence, issue des calcaires marmorisés de l'Orradag, qui a été captée auprès du village d'Irviz pour faire tourner les turbo-alternateurs d'une centrale destinée à fournir la force motrice à la ville industrielle d'Eregli. Dès une très haute antiquité ce site, plein de fraîcheur, de bruits d'eau et de feuillages, était fréquenté par les populations hittites puisqu'on y vient toujours pour contempler un bas-relief de facture assyrienne et représentant Warbalawa, roi de Tuvanuva, en adoration devant le dieu Tarhum, dispensateur de l'eau précieuse qui fait mûrir, au pied de la montagne riche en neige, les champs de blé et les raisins des treilles ; un épi et une grappe symbolisent dans la main du dieu les dons de l'eau bienfaisante dans ces pays de climat semi-aride.

De Mersin à Silifké, la Cilicie Trachée offre un riche terrain d'observations aux karstologues et aux spéléologues avec ses longues bandes de travertins qui, au pied des plateaux calcaires, retombée du Taurus vers la mer, prolongent vers le Sud-Ouest les énormes accumulations de calcite, creusées de canyons et de bassins fermés entre Adana et Ceyhan. Nous eûmes le loisir de parcourir de vastes champs de lapiès, parsemés de blocs rocheux perforés en tous sens, au milieu de chênes kermès et d'asphodèles. Des gouffres et des grottes s'ouvrent au milieu de ces âpres reliefs comparables à nos causses les plus rugueux. Le tout a été modelé sans doute au cours de l'ère quaternaire, en un laps de temps géologiquement assez court si l'on en juge par la rapidité avec laquelle ont été creusées, depuis moins d'un millénaire, les rainures qui altèrent les murailles des châteaux-forts en ruine d'Anamur et de Korigos. Dans la masse des sédiments miocènes de Kanlidivane, au milieu des ruines de Pompéiopolis et de Neapolis, s'ouvrent les gouffres du Paradis et de l'Enfer. On peut descendre dans le premier par des sentiers fort raides jusqu'auprès des murs d'une chapelle ; mais il faut des cordes pour atteindre le fond du second, exploit réalisé récemment par Temucin Aygen. Ces gouffres sont en quelque sorte des "Padiracs», taraudés dans les strates calcaires par corrosion sans doute, mais également par des effondrements provoqués par les attaques simultanées des cours d'eau souterrains et des infiltrations d'origine subaérienne. L'une de ces rivières hypogées, celle qui passe dans le gouffre du Paradis et qui draine aussi la grotte de Narlijutu, située à $300 \mathrm{~m}$. vers l'Ouest, reparaît sur le rivage, dans la crique de Karacol, en une résurgence sous-marine qui refoule de ses eaux douces vers le large les eaux salées de la Méditerranée. 
A Gilindiré, sur la route de Silifké à Anamur, une autre source vauclusienne était depuis longtemps captée pour faire tourner la roue à aubes d'un moulin à blé. Des travaux en cours vont permettre d'en élever les eaux à une soixantaine de mètres afin de les répandre ensuite par gravité dans la plaine voisine où des cultures de primeurs et de coton remplaceront les marécages à roseaux et les médiocres récoltes de céréales. Plus loin, vers l'Ouest, au-delà d'Alanya, les sources de la Manavgat se brisent sur des bancs de poudingue, dans un cadre de fraîche verdure, bien fait pour attirer les touristes. La rivière, qui n'a guère plus de $80 \mathrm{~km}$. de longueur, doit drainer, si l'on en juge par son abondance, un vaste massif calcaire jurassique et crétacé à l'intérieur du Taurus central. Des résurgences, au débit très variable, lui apportent également une alimentation très appréciable et que l'on souhaiterait utiliser plus largement comme force motrice et pour l'irrigation. Un barrage est en construction en amont des chutes pour créer un réservoir qui servira à double fin : courant électrique et eau pour les cultures (Fig. 1).

Ce sont également des sources vauclusiennes qui donnent naissance au cours d'eau qui a été capté au Nord-Ouest d'Antalya pour animer la centrale hydroélectrique destinée à fournir du courant à la ville. Avant son aménagement, ce fleuve côtier se perdait dans l'énorme masse de travertins qui s'est accumulée au Nord d'Antalya, au débouché de la coupure qui permet d'atteindre le lac d'Egridir et les hauts plateaux d'Afyon. On pouvait cependant l'apercevoir au fond du gouffre de Varsak où il formait un lac. Puis il reparaissait à la sortie d'une grotte, sous les travertins de Dudençay; de là il s'écoulait vers la mer en faisant tourner des moulins et en irriguant la plaine au pied de Pergé. Des eaux aussi précieuses ont failli être perdues pour les agriculteurs de la région quand elles ont été captées pour la centrale de Kepez. Une judicieuse intervention du géologue Temucin Aygen a permis de les récupérer en les ramenant dans leur ancien lit par un canal qui les prend à la sortie de l'usine et, par un cours sinueux, les conduit au-dessus de l'ancienne source, à la surface des bancs de travertins, d'où elles tombent en une magnifique cascade dans leur ancien lit. Ces divers travaux, choisis parmi beaucoup d'autres, montrent quel intérêt l'administration turque porte aux richesses du pays en houille blanche et en eau d'irrigation et, par contre-coup, aux recherches, parfois si fructueuses et toujours passionnantes, des spéléologues d'Istanbul et d'Ankara.

Nous ne voudrions pas terminer ce bref compte-rendu sans signaler encore à nos collègues quelques autres phénomènes karstiques d'Anatolie qui permettent de confirmer ou d'infirmer les lois du relief dans 
les roches calcaires. Ainsi, autour du rocher d'Alanya, que couronne la forteresse seldjoukide, les grottes des Pirates, des Amoureux, et la grotte à Phosphore, outre leurs admirables jeux de lumière dans les eaux marines, montrent comment le récent mouvement eustatique de la Méditerranée a envahi les anfractuosités des rochers calcaires sans y apporter encore de modifications sensibles. Ces cavités sont à rapprocher de Glyphada en Péloponnèse, de la grotte d'Emeraude à Amalfi et de bien d'autres où a pénétré la transgression flandrienne. Au pied des mêmes rochers s'ouvre la curieuse caverne de Damlatas où, grâce aux travaux du Docteur H. Sipahioglu, les malades des bronches, les asthmatiques, éprouvent un soulagement à leurs maux après y avoir séjourné à plusieurs reprises durant quelques heures.

Si nous n'avions pu visiter les travertins d'Adana, il nous fut possible au contraire de parcourir ceux d'Antalya qui s'étendent sur $600 \mathrm{~km} .{ }^{2}$, au Nord de la ville et au débouché de la pincée où s'infléchissent vers le Nord les plissements du Taurus occidental et du Taurus central. Composés de calcite déposée autour d'une abondante et étrange végétation de prêles, de roseaux et de polypiers géants (nous en avons photographié un de 2,50 m. de haut, Fig. 4), ils s'étagent en plusieurs niveaux correspondant sans doute à des variations du niveau marin, ou à des exhaussements du continent. Nulle part au monde

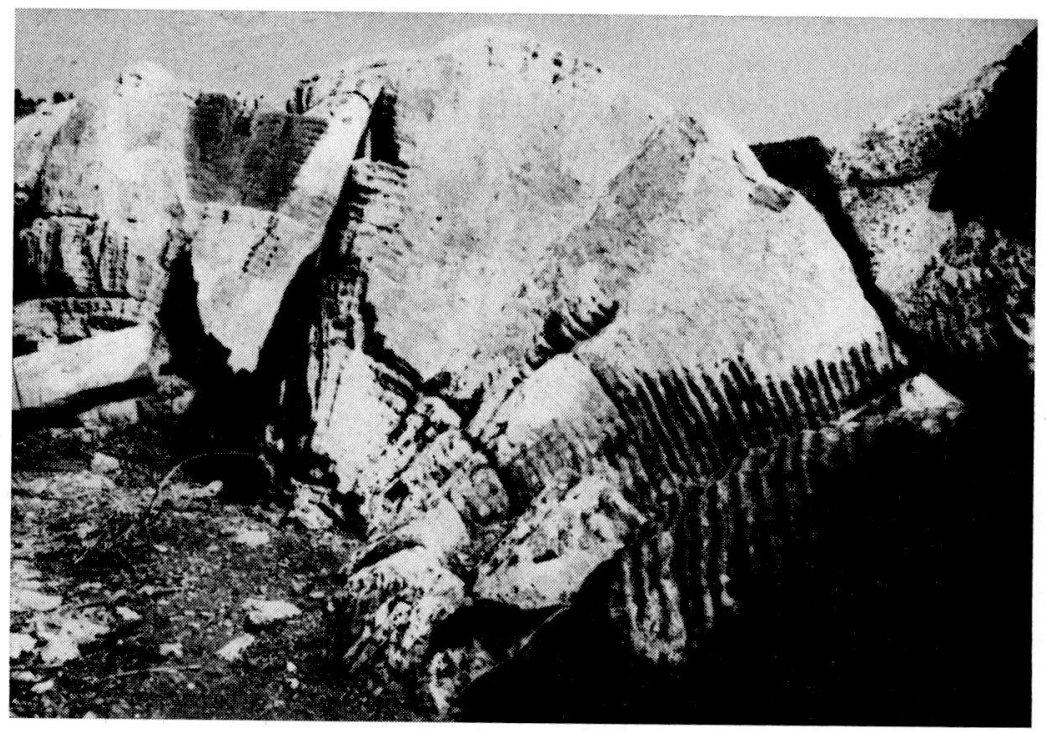

Fig. 4. Polypier géant (2,50 m.) dans les travertins d'Antalya. 
sans doute, il ne serait aussi facile et aussi probant de mettre en valeur ces divers mouvements, ni d'analyser les conditions dans lesquelles se forment ces dépôts de $\mathrm{CO}_{3} \mathrm{Ca}^{4}{ }^{4}$ )

$\mathrm{Au}$ cours du retour en avion d'Antalya à Istanbul par Afyonkarahissar, nous survolâmes les poljés dont nous avait entretenu le Professeur Ismaïl Yalcinlar lors de la conférence de Beyazit. Quelques uns sont encore occupés par des lacs (Kovada Göl, Egridir Göl) ; d'autres au contraire sont asséchés, au moins temporairement, à Anamas, à Candir et à Buçak. Tous reflètent dans leurs formes les longs alignements des plissements du Taurus. Dans cette région, la Turquie possède donc un admirable terrain d'exploration pour les phénomènes karstiques ; nos collègues d'Anatolie y ont déjà réalisé de fort précieuses recherches ; mais il reste encore beaucoup à faire pour élucider les problèmes posés par les vastes et curieuses dépressions fermées de toute cette zone montagneuse.

Enfin, au-delà d'Afyon, au Sud-Est de Kutahya, nous avons aperçu un curieux relief qui nous a paru d'origine karstique ; il est composé de collines arrondies taraudées par d'énormes cupules régulières, comme en offrent certains polypiers. Mais ici les dimensions de ces cavités circulaires paraissent atteindre plusieurs dizaines de mètres. La photographie que nous en avons prise permettrait peut-être à nos confrères de la Société Spéléologique de Turquie de nous renseigner à ce sujet, d'expliquer ce comportement d'un massif sans doute calcaire, qu'ils connaissent probablement déjà d'une manière très exacte. Nous nous en remettons donc à leur appréciation.

Telles sont les observations, les remarques et les réflexions tirées du rapide périple accompli à travers la péninsule d'Asie Mineure. Très bien conçu par le géologue Temucin Aygen, il nous a permis de confronter la réalité d'un milieu extrêmement divers avec les communications de la Conférence d'Istanbul et avec les reliefs karstiques de Grèce et de France. Grâce à des rapprochements aisément réalisables, tels ceux dont nous fit part le Géographe Ahmed Ardel dans l'amphithéâtre de son Institut de Beyazit, la Spéléologie et la Karstologie, étroitement unies, peuvent progresser aussi bien dans les voies de la recherche fondamentale que dans celles des applications

4) Voir X. de Planhol : Position stratigraphique et significations morphologiques des travertins subtauriques de l'Anatolie sud-occidentale. Actes du IV ${ }^{\circ}$ Congrès international du Quaternaire. 1953. 
pratiques. C'est vers cette double perspective que s'orientent nos collègues de Turquie ; on ne peut que les en féliciter et, en les remerciant de leur aimable accueil, leur souhaiter les fructueux résultats que méritent leurs efforts et leur compétence.

\section{RÉSUMÉ}

Réunie à l'instigation du Docteur géologue Temucin Aygen, la Conférence internationale de Spéléologie et de Karstologie d'Istanbul, ou participèrent une vingtaine de savants étrangers, se déroula d'abord à l'Université Beyazit de Stamboul. Durant trois jours, communications et discussions animèrent les séances, entrecoupées d'excursions autour du Bosphore.

Ensuite, durant deux semaines, les membres de la Conférence effectuèrent un voyage d'étude à travers l'Anatolie, par Ankara, Konya, Mersin, Antalya, Burdur, Izmir, Bursa et Istanbul. Ils eurent ainsi l'occasion d'explorer les principaux phénomènes karstiques de Turquie: obrouks de Konya, travertins de Yerköpru et d'Antalyà, grottes de la région de Mersin, sources vauclusiennes d'Irviz et de la Manavgat, etc. L'intérêt de ces accidents du karst anatolien est indéniable ; outre les problèmes scientifiques qu'ils posent, ils représentent une grande valeur économique soit comme centres touristiques, soit comme producteurs d'énergie électrique et sources d'irrigation.

\section{SUMMARY}

Arranged by geologist Dr. Temucin Aygen, the International Conference of Speleology and Karstology at Istanbul, with the participation of about twenty foreign scholars, opened first at Beyazit de Stamboul University. For three days papers and discussions enlivened the sessions, broken by tours around the Bosporus.

During the following two weeks the members of the Conference took a field trip across Anatolia, through Ankara, Konya, Mersin, Antalya, Burdur, Izmir, Bursa, and Istanbul. They thus had the opportunity to investigate the principal karstic phenomena of Turkey - the Konya obrouks, travertines of Yerköpru and Antalya, caves of the Mersin region, vauclusian springs of Irviz and Manavgat, and so forth. The interest of these occurrences of Anatolian karst is unquestionable-in addition to the scientific problems they pose, they represent a great economic value either as tourist centers or as producers of electric energy and sources of water for irrigation. 